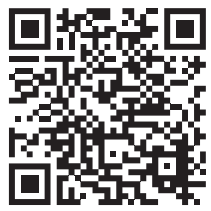

Palabras clave: Coronavirus, SARSCoV-2, atención de enfermería, prevención y control, prevención y mitigación.

Keywords: Coronavirus, SARSCoV-2, nursing

care, prevention and control, prevention and mitigation.

* Departamento de Investigación en Enfermería del INCICh. Vocal de Enfermería de la Sociedad Mexicana de Cardiología.

¥ Directora de

Enfermería del INCICh. Miembro del Comité de Enfermería de la Sociedad Mexicana de Trombosis y Hemostasia, A.C.

$\S$ Departamento de Enseñanza de Enfermería del INCICh. Miembro del Comité de Enfermería de la Sociedad Mexicana de Trombosis y Hemostasia, A.C. " Servicio de Hemodinámica del INCICh. Vocal de Enfermería de la Sociedad de Cardiología Intervencionista de México.

\title{
Enfermería ante el COVID-19, un punto clave para la prevención, control y mitigación de la pandemia
}

\author{
Nursing before COVID-19, a key point for the prevention, \\ control and mitigation of the pandemic \\ Julio C Cadena-Estrada, ${ }^{*}$ Sandra S Olvera-Arreola, ${ }^{\ddagger}$ Liliana López-Flores, ${ }^{\S}$ Elvia Pérez-Hernández,, \\ Gabriela Lira-Rodríguez, "Noé Sánchez-Cisneros, ${ }^{* *}$ Martha M Quintero-Barrios ${ }^{\ddagger}$
}

\section{RESUMEN}

El brote de SARS-CoV-2 o COVID-19 originado en China ha alcanzado a México. No obstante, la comunidad científica, entre ellas enfermería, ha generado de manera vertiginosa evidencia que puede ayudar a tomar decisiones en la atención de la población afectada. El presente documento tiene la finalidad de describir la experiencia de enfermería ante el COVID-19 como un punto clave para la prevención, control y mitigación de la pandemia. Con base en las recomendaciones emitidas por la Secretaría de Salud, la evidencia clínica y los recursos disponibles en las instituciones de salud se ha iniciado y mantenido las medidas de prevención de la enfermedad tanto en la comunidad como en las instituciones de salud. La reconversión de los hospitales y los protocolos de atención adaptados a nuestro contexto están tratando de fortalecer el control y mitigación de la enfermedad.

\section{ABSTRACT}

The SARS-CoV-2 or COVID-19 outbreak originated in China has reached Mexico. However, the scientific community, including nursing, has generated vertiginous evidence that can help make decisions in the care of the affected population. The purpose of this study is to describe the nursing experience before COVID-19 as a key point for the prevention, control and mitigation of the pandemic. Based on the recommendations issued by the Ministry of Health, the clinical evidence and the resources available in the health institutions, the disease prevention measures have been initiated and maintained both in the community and in the health institutions. The reconversion of hospitals and care protocols adapted to our context are trying to strengthen the control and mitigation of the disease.

\section{PANDEMIA DE COVID-19: SU LLEGADA A MÉXICO}

$\mathrm{E}^{n}$ n el último día de diciembre de 2019 la Comisión Municipal de Salud y Sanidad China informó sobre 27 casos de neumonía de causa inespecífica, con el antecedente de exposición común a un mercado de mariscos, pescado y animales vivos en la ciudad de Wuhan. El 7 de enero de 2020, las autoridades sanitarias identificaron como agente causante un nuevo tipo de virus de la familia Coronaviridae que posteriormente fue llamado SARS-CoV-2 o COVID-19, cuya secuencia genética fue compartida el 12 de enero. En el brote, la transmisión intrafamiliar fue muy frecuente, lo que ocasionó una rápida propagación a la comunidad y una alta transmisión intrahospitalaria en trabajadores sanitarios de los hospitales.

Dada la comunicación estrecha que tiene China con otros países, la enfermedad se propagó a la región asiática, y el 21 de enero el Centro para el Control y la Prevención de Enfermedades (CDC) de los Estados Unidos de América informó el primer caso confirmado importado con el antecedente de viaje a Wuhan, China. Tal ha sido su expansión de la enfermedad que el 11 de marzo la Organización Mundial de la Salud la declaró como una pandemia. Desde el brote, las autoridades de la Secretaría de Salud de México comenzaron a emitir recomendaciones para quienes regresaban de viajes de China y para quienes habían convivido con casos sospechosos o confirmados de COVID-19, además activó la Unidad de In- 
" Departamento de

Mejora Continua del Cuidado de Enfermería del INCICh. Ex Vocal de Enfermería de la Asociación Mexicana de Cardiopatías Congénitas, A.C. ** Departamento de Gestión Operativa del INCICh.

枺Departamento de Gestión Clínica del INCICh. Ex Vocal de Enfermería de la Asociación Mexicana de Cardiopatías Congénitas, A.C.

Instituto Nacional de Cardiología «Ignacio Chávez» (INCICh). Ciudad de México, México.

Recibido: 20/04/2020 Aceptado: 29/04/2020 teligencia Epidemiológica y Sanitaria, logrando identificar durante el mes de enero y hasta el 27 de febrero 22 casos negativos. Sin embargo, como era de esperarse, la enfermedad llegó a México por importación al confirmarse el 28 de febrero el primer caso positivo de COVID-19. Al 16 de abril se han confirmado 6,297 casos y desgraciadamente 486 han fallecido. ${ }^{1}$

\section{ESTRATEGIAS DE PREVENCIÓN EN ENFERMERAS ROTANTES EN EL ÁREA HOSPITALARIA}

El impacto de la pandemia por COVID-19 a nivel mundial ha venido a mermar todos los ámbitos de la sociedad y el ámbito educativo no es la excepción. La mayoría de los gobiernos de todo el mundo han dado instrucciones para cerrar temporalmente las instituciones educativas en un intento por contener la propagación de esta pandemia.

Una investigación realizada por el Tecnológico de Monterrey pone de manifiesto las afectaciones a la educación en México, señala una serie de retos que el sistema educativo está enfrentando ante la contingencia, de manera puntual que la mayor parte de los planteles (a todos los niveles) no están preparados para dar una buena educación a distancia, la mayoría de los docentes a nivel básico no han sido capacitados para enseñar de manera remota y, aunado a esto, no todos los estudiantes tienen la posibilidad de llevar clases a distancia, puesto que no cuentan con acceso a internet. Como una medida para subsanar un poco el cierre de las escuelas, la Secretaría de Educación se ha apoyado en educación a través de los canales de televisión (11 y 22, específicamente) para niveles de preescolar, primaria y secundaria; esto es una buena opción, pero el alcance es poco aunado a que debe existir un compromiso por parte de los padres de familia para que el estudiante recurra a esta opción de manera puntual y con el sesgo de que en el momento no existe un docente que vaya acompañando al alumno en las lecciones que se van mostrando.

Para niveles medio superior y superior las opciones en línea son más comunes, puesto que se llevan a cabo a lo largo de los ciclos escolares con apoyo de diferentes plataformas, pero existen carreras en las que es completamente necesaria la opción presencial para desarrollar habilidades que no se obtienen fácilmente en línea, un ejemplo de ellas son las carreras que competen al ámbito de la salud, en donde la práctica clínica es la única opción para adquirir el aprendizaje y las competencias necesarias para la vida laboral.

En las instituciones de salud como el Instituto Nacional de Cardiología «lgnacio Chávez» ( $\mathrm{INClCh}$ ), se tiene una amplia rotación durante todo el año de médicos y enfermeros de pregrado y postgrado en práctica clínica, que ante esta contingencia hubo la necesidad de restringir su entrada al Instituto para salvaguardar su integridad. Esta decisión se tomó con base en indicaciones de la Dirección de Enseñanza a partir del 17 de marzo, por lo que hubo comunicación inmediata con las instituciones que se encontraban rotando y con aquéllas que iniciarían durante los meses de marzo, abril y mayo, con la comprensión de las mismas y quedando en acuerdo que las autoridades de cada institución aplicarían la medida inmediatamente y establecería actividades en línea para que los alumnos no perdieran continuidad en su aprendizaje. Sin embargo, como se ha mencionado, estas actividades en línea coadyuvan al aprendizaje, pero limitan el desarrollo de competencias, las cuales se adquieren al estar en el ámbito real ante los pacientes.

\section{RECURSOS MATERIALES Y HUMANOS, UNA ADQUISICIÓN INTELIGENTE CONTRA LA PANDEMIA}

En esta época en que el entorno se vuelve tan dinámico y el cambio constante se torna complejo y lleno de incertidumbre, es imperante para las instituciones de salud hacer un alto y comenzar con la gestión del recurso humano de forma estratégica, pero sobre todo eficaz y eficientemente. El recurso humano es el eje rector de la operación de las instituciones, por lo cual los directivos se deben enfocar en la capacitación y el conocimiento de los colaboradores, ya que esto explicará el resultado, encaminado al éxito, el control y la mitigación de la pandemia de COVID-19.

De acuerdo al decreto emitido el día 23 de marzo en el Diario Oficial de la Federación, donde se establece que «se permite el trabajo 
en casa a los servidores públicos mayores de 60 años, a personas con discapacidad, mujeres embarazadas y con enfermedades crónicas», las instituciones de salud analizaron el panorama de la operatividad en función de la ocupación hospitalaria, de tal forma que se organizó a los colaboradores activos, por turno y por servicio, para que se mantuvieran en resguardo domiciliario, entre 30 y $40 \%$, garantizando la atención con seguridad para el paciente. Esta decisión tomada por los directivos de la institución y organizada por las supervisoras y jefes de enfermería ha sido flexible y ha permitido disminuir el riesgo de contagio, pero sobre todo ha permitido al personal de enfermería mantener un equilibrio entre la vida personal y laboral, mismo que se ve reflejado en la operatividad en servicio y en la capacidad de respuesta ante esta pandemia.

\section{LA RECONVERSIÓN DE LA ATENCIÓN DEL PACIENTE CON COVID-19: EL CUIDADO CLAVE DE ENFERMERÍA}

El profesional de enfermería es el elemento clave en el cuidado del paciente cardiópata o no cardiópata con COVID-19 en las instituciones de salud. Todo debe estar planificado, desde el primer contacto en el triaje hasta prácticamente el egreso por mejoría o defunción.

La participación del personal de enfermería no es ajeno en ninguna de las estaciones o etapas de atención al paciente con COVID-19; para la participación del personal de enfermería en esta contingencia se planteó proteger en todo momento al equipo de trabajo y asegurar la calidad del cuidado bajo premisas aprendidas e inferidas de los procesos ya establecidos y de las experiencias de otras instituciones.

\section{Protección del equipo de salud}

1. Se colaboró con el equipo multidisciplinario para establecer las estaciones de tamizaje y atención con rutas específicas de tránsito de pacientes sospechosos o confirmados de COVID-19 y del resto de usuarios de la institución (internos y externos).

a. Filtros en todas las entradas: personal de seguridad y de enfermería realizan una medición de la temperatura con técnica de rayo infrarrojo y un test para detectar signos y síntomas respiratorios a todos los usuarios de la institución. En caso de encontrar alteraciones según los resultados, se refieren a la siguiente estación, triaje I.

b. Tamizaje o triaje I: un equipo de salud realiza la valoración de signos y síntomas, principalmente de fiebre, tos seca, estornudos, malestar general, astenia, cefalea y dolor de garganta, para identificar y discriminar los caszos sospechosos, identificar el nivel de sintomatología y repercusión clínica. Se refieren a casa con indicaciones médicas y datos de alarma a personas con síntomas leves o moderados (de aquí la gran importancia de la teleenfermería y telemedicina). En caso de datos de insuficiencia respiratoria se refieren al triaje II y con datos de descompensación cardiopulmonar al consultorio.

c. Triaje respiratorio o II: médicos y enfermeras de primera intención realizan una valoración física focalizada, establecen acceso venoso, toman muestras sanguíneas, hisopado faríngeo, la decisión contempla egreso a casa o confinamiento hospitalario.

d. Consultorio: denominado así por ser uno de los consultorios de atención cardiovascular, elegido por su infraestructura (proximidad, espacio y aislamiento). En esta estación se otorga atención crítica con monitoreo hemodinámico y administración de medicamentos más cercano, la decisión contempla egreso a casa o a confinamiento hospitalario.

e. TIC COVID: denominado así por ser la terapia intensiva convertida ahora para la atención del paciente sospechoso o confirmado con COVID-19, el nombre de la estación indica el grado de atención para este grupo de pacientes.

f. Piso 8 COVID: piso de atención destinado a la atención en convalecencia y rehabilitación del paciente. 
2. Se deben adecuar las estaciones o áreas de atención bajo las siguientes premisas:

a. Establecimiento de rutas de circulación de personal, pacientes y desechos (diferentes en todo momento o si la infraestructura no lo permite con delimitaciones físicas y visuales).

b. Diseño e implementación de esclusas de entrada y de salida (instalación y retiro del equipo de protección personal).

c. Equipo de protección personal (EPP) semaforizado por el grado y tiempo de exposición, para la optimización del recurso, en bajo, mediano y alto riesgo (verde, amarillo y rojo, respectivamente).

3. La capacitación de todas las actividades sustantivas para el otorgamiento del cuidado, con énfasis en la instalación y retiro de EPP.

\section{Gestión del cuidado}

El cuidado gira en torno a la prevención de contagio a través del uso adecuado del EPP en las áreas COVID, higiene de manos, exposición a aerosoles y excretas y el mínimo del talento humano para la atención, de tal manera que se han protocolizado y adecuado los siguientes procesos:

1. Traslado intrahospitalario (cerrado desde el triaje a la UCl y/o hospitalización, restricción de visitas y la sanitización del área simultánea al procedimiento).

2. Manejo de excretas y los utensilios con tal fin.

3. Baño de paciente (paño húmedo).

4. Cambio de posición a decúbito prono (con tres y cinco personas).

5. Manejo y traslado de muestras de laboratorio.

6. Alimentación enteral.

7. Amortajamiento.

8. Egreso del paciente por mejoría.

Sin duda el resto del cuidado no sufre modificaciones. Dado la cadena de transmisión de la enfermedad, es pertinente comentar que el resto de los procedimientos (metas internacionales, estándares de calidad y cuidados generales) no deben sufrir modificaciones y el manejo de ropa y desechos se realiza como lo recomendado en las normas oficiales mexicanas.

\section{Consideraciones especiales en el entorno del cuidado}

La dignidad humana de todos se debe garantizar, para ello no se debe atrever (dentro del marco jurídico, administrativo, laboral y social) a innovar aún más, por mencionar al menos cuatro aspectos:

1. En el contexto que aquel paciente contagiado no recibe visitas por las barreras sanitarias, se desarrolla un proceso para que vía remota pueda realizar videollamadas con sus seres queridos.

2. La defunción es un egreso posible en esta contingencia, no sólo con fines jurídicos como la identificación del cadáver, sino desde el punto de vista humano, se desarrollaron bolsas de cadáver con una parte transparente para que la familia pueda despedirse bajo su cosmovisión.

3. Para los registros, con su importancia jurídica y administrativa, se estableció un repositorio de archivos para la consulta y acceso por cualquier causa o motivo, mientras se cumple la cuarentena del papel expuesto en el área COVID.

4. Se diseñan y adecuan áreas para que el equipo de trabajo (todos, desde personal de intendencia hasta el médico líder de la jornada) tenga espacios de higiene mental que soslayen el cansancio de una jornada breve (en comparación con el tiempo de jornada), pero con intensa carga de trabajo.

En estos momentos el valor agregado a esta contingencia ha sido el reforzamiento de la fraternidad con disciplinas y áreas afines al cuidado, donde los protagonistas no sólo son las disciplinas del área de la salud, también son aquéllas cuyo trabajo son el pivote para desencadenar procesos, y con la salvedad que en este momento se escape alguna, es todo aquel personal afanador, seguridad, lavandería y mantenimiento, sin estas figuras no sería posible el contener contaminaciones, transmisiones, otorgar cuidados y asegurar las áreas. 



\section{Figura 1:}

Protocolo para salas de hemodinámica ante el brote de COVID-19, antes, durante y después del procedimiento intervencionista.




El cuidado no es ajeno al entorno local y global, algunos retos por mencionar es la gestión y eficiencia de insumos (rapiña, robo, subutilización, escasez, presupuesto) y asegurar el cuidado de calidad con el talento humano disponible (grupos vulnerables en resguardo, incapacidades por diversos motivos, entre ellas contagios con COVID-19, solicitud de prestaciones laborales por miedo y ansiedad), alusión a la vocación de servicio, limar asperezas, convencimiento de realizar actividades de otras áreas.

No hay mejor situación en el año de enfermería que el enfrentarnos a esta pandemia, es el momento y la oportunidad de demostrar con hechos el compromiso social, la voluntad personal y la esencia profesional. Es momento de que el mundo vea lo que somos como profesión y como ese engrane imprescindible del sistema de salud. De forma personal convocamos a hacernos visibles en todos lados.

\section{PROTOCOLO PARA SALAS DE HEMODINÁMICA ANTE EL BROTE DE COVID-19}

La pandemia de COVID-19 ha llegado a modificar los procesos de trabajo de todo el personal profesional y no profesional del área de la salud, y el servicio de hemodinámica no está exento al ser un departamento donde es necesaria la participación de un equipo multidisciplinario para atender a los pacientes cardiópatas en un tiempo que varía de 30 minutos a cinco horas dependiendo de la especialidad. Es importante considerar que «no hay emergencias en una pandemia», pero es indispensable prever los escenarios de posibles complicaciones y asignar un rol a cada integrante; la experiencia desarrollada por cada profesional permite compartir con sus compañeros el conocimiento, las habilidades y las competencias adquiridos, proporcionando certidumbre en el manejo de situaciones críticas como las que estamos viviendo actualmente. Ante esta pandemia ha sido preciso desarrollar protocolos que permitan comunicar de manera gráfica y específica el procedimiento intervencionista por el aislamiento estricto para los pacientes con COVID-19, tanto antes, durante y después del procedimiento con el EPP especializado. Todo inicia activando los códigos descritos en la Figura 1.

\section{REFERENCIAS}

1. Secretaría de Salud [Internet]. Comunicado Técnico Diario Nuevo Coronavirus en el mundo (COVID-19). México: Subsecretaría de Prevención y Promoción de la Salud; 2020. Disponible en: https://www.gob. $\mathrm{mx} /$ salud/documentos/informacion-internacional-ynacional-sobre-nuevo-coronavi- rus-2019-ncov

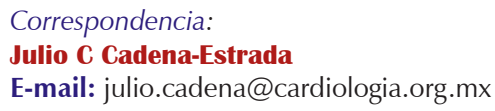

\section{RESPONSABILIDADES ÉTICAS}

Protección de personas y animales. Los autores declaran que para esta investigación no se han realizado experimentos en seres humanos ni en animales.

Confidencialidad de los datos. Los autores declaran que han seguido los protocolos de su centro de trabajo sobre la publicación de datos de pacientes.

Derecho a la privacidad y consentimiento informado. Los autores declaran que en este artículo no aparecen datos de pacientes. 